\title{
Exkurs: Entscheidungsfindung zur Organspende in der Pädiatrie
}

\author{
Klaus Michael Lücking
}

Eine Organspende bedarf - wie jeder ärztliche Eingriff - einer ärztlichen Indikation, welche mit dem Patientenwillen in Einklang zu bringen ist. Der Deutsche Bundestag hat mit dem Beschluss des „Gesetzes zur Stärkung der Entscheidungsbereitschaft bei der Organspende“ die Zustimmung des Patienten resp. seiner Stellvertreter zur postmortalen Organspende ausdrücklich unterstrichen - und eine „Zustimmung durch Schweigen“ i.S. der sog. Widerspruchslösung verworfen. Die grundlegende Forderung nach diesem Einklang von medizinischer Indikation und Patientenwillen findet sich auch in den Empfehlungen zur Entscheidungsfindung über das übergeordnete intensivmedizinische Therapieziel (Janssens et al. 2013; Neitzke et al. 2016). Die neue Richtlinie der Bundesärztekammer zur Spendererkennung greift die langjährig erhobene Forderung nach Stärkung der Patientenautonomie im gesamten Organspendeprozess auf (Schöne-Seifert et al. 2011; Hahnenkamp et al. 2016): Die Richtlinie fordert nun die Erforschung des Patientenwillens in engem Austausch mit den Patientenstellvertretern vom Moment des Therapiezielwechsels bei infauster Prognose über die Phase der „organprotektiven“ Intensivtherapie und Feststellung des Irreversiblen Hirnfunktionsausfalls (IHA) bis hin zur schlussendlichen Organentnahme-Operation (Bundesärztekammer 2020).

Doch welche Auswirkungen hat dieser Paradigmenwechsel, insbesondere die Betonung des Patientenwillens, auf die Entscheidungsfindung bei pädiatrischen, gar neonatologischen Patienten? Die Entscheidungsfindung für oder gegen eine Organspende offenbart auch das grundlegende Autonomie- 
dilemma in der Behandlung kindlicher Patienten: Die Autonomie von Kindern ist häufig eingeschränkt durch ihren Entwicklungsstand (welcher nicht zwingend mit dem chronologischen Alter korreliert), durch etwaige Behinderungen und auch das aktuelle Krankheitsbild. Eltern fungieren hier häufig als „Proxy-Entscheider“, wenngleich diese die Wünsche und Willensbekundungen ihrer Kinder in ihre Entscheidung einfließen lassen sollen. Das UK Donation Ethics Committee empfiehlt, spätestens Adoleszenten ab dem 12. Lebensjahr ein gewichtiges Mitspracherecht (und damit einen „mutmaßlichen“ Willen) in der eigenen Therapieplanung und bei diesbezüglichen Entscheidungen einzuräumen (Commitee on Bioethics 2013). Dieses könne sogar - je nach individuellem Entwicklungsstand - bereits in der Altersgruppe der 8- bis 11-Jährigen erwogen werden. Dejure gestattet das deutsche Transplantationsgesetz Jugendlichen ab dem 16. Lebensjahr eine dezidierte Entscheidung für eine Organspende; ab dem 14. Lebensjahr kann ein Jugendlicher bereits vorsorglich gegen eine Organspende votieren (Bundesministerium für Justiz und Verbraucherschutz 2021).

Verschiedene auch pädiatrische Fachgesellschaften und nationale Aktionspläne fordern, die Organspende als routinemäßigen Bestandteil der Intensivtherapie am Lebensende wahrzunehmen und angemessen zu evaluieren (ESPNIC; Kotloff et al. 2015; Nakagawa et al. 2018). Doch welches „Organspendepotential“ ist auf pädiatrischen Intensivstationen (PICU) zu erwarten? Die Analyse von ca. 10.00o PICU-Aufnahmen in 5 tertiären US-amerikanischen pädiatrischen Zentren zeigte eine Gesamtmortalität von 2,4\%: 70\% der Kinder starben nach einem Verzicht auf resp. nach Abbruch der Intensivtherapie (2/3 hiervon mit „Do-not-attempt-resuscitation“(DNAR)-Vorgabe); bei lediglich 16\% wurde die Diagnose eines IHA gestellt (Burns et al. 2014). Auch die zugrundeliegenden Diagnosen (von onkologischen Krankheitsbildern bis hin zu Stoffwechselerkrankungen und anderen, gelegentlich uncharakterisierten Krankheitsbildern als relevante Kontraindikationen) schränkt die Auswahl potentieller kindlicher Organspender ein (Brierley u. Hasan 2012). Lediglich knapp 3\% (53/1.837) der Organspenden des Eurotransplant-Raumes wurden 2020 auf pädiatrischen und neonatologischen Intensivstationen realisiert (Eurotransplant 2021). Ähnliche Zahlen aus den USA, Australien und dem Vereinigten Königreich in den vergangenen Jahren belegen, dass weltweit die Organspende ein rares Ereignis auf der pädiatrischen und neonatologischen Intensivstation darstellt (Gelbart 2017; Hawkins et al. 2018).

Gleichzeitig haben die ärztlichen und pflegerischen Behandler/Bezugspersonen in den Entscheidungsprozessen zum übergeordneten Therapieziel am Lebensende (und damit auch zur Option der Organspende) einen gewichtigen Stellenwert. Stabile tragfähige Entscheidungen zur Organspende werden maßgeblich davon beeinflusst, ob die Frage nach der Organspende aus 
den Intensivteams herausgestellt wird (Rodrigue et al. 2008). Eine strikte Rollentrennung von Intensiv- versus Organspendeteam („de-coupling“) ist also auf der pädiatrischen Intensivstation nicht hilfreich für die Entscheidungsfindung. Umso wichtiger erscheint ein interprofessionelles Herangehen unter frühzeitigem Einbezug eines (inhouse-) Organspendeteams zu sein: Dies kann helfen, Unsicherheiten und Friktionen bzgl. einer möglichen Organspende, aber auch ganz konkret zum Umfang und zur Dauer einer organprotektiven Intensivtherapie zu verbalisieren und zu klären (Brierley u. Shaw 2016; Neitzke et al. 2019).

Sicherheit in der Gesprächsführungskompetenz können bekannte Formate (wie SPIKES u.a.) vermitteln (Jöbges et al. 2019). Mindestens ebenso wichtig erscheint es, die eigene Haltung zur Organspende zu reflektieren, um in einen ergebnisoffenen Dialog mit der Familie einzutreten (Kentish-Barnes et al. 2017). Zeit und ggf. wiederholte Gesprächsangebote spielen in einer familienzentrierten Annäherung an die Entscheidung eine zentrale Rolle, ebenso wie eine Fokussierung auf die wichtigen familiären Gesprächspartner (ggf. unter aktivem Einbezug von Meinungsbildnern, welche primär nicht „mit am Tisch sitzen“) und nolens volens eine klare Ergebnisorientierung. In einer zunehmend diversifizierten Gesellschaft empfiehlt es sich, die religiösen, spirituellen und weltanschaulichen Perspektiven der Familie, auch ihre eigenen Bilder zur Organspende, zu erfragen und in den Entscheidungsprozess zu integrieren. Nicht selten wird die Entscheidung für eine Organspende sowohl in der Akutsituation als auch in Rückschau als „erleichternd, weil sinnstiftend“ von den Eltern und ggf. auch anderen Familienmitgliedern wahrgenommen und bewertet. Deshalb erscheint es nicht unlauter, mit den Eltern Neugeborener und kleiner Kinder (also von Kindern, denen ein autonomer Patientenwille nicht zwingend unterstellt werden darf) die eigenen Wünsche als „Zurückbleibende“ zu erforschen. Der familienzentrierte Weg zur Entscheidung über die Organspende als Option am Lebensende sollte auch einschließen, in der Zukunft für eine Reflexion der akut getroffenen Entscheidungen zur Verfügung zu stehen (KentishBarnes et al. 2019).

\section{Literatur}

Brierley I, Hasan A (2012) Aspects of deceased organ donation in paediatrics. Br | Anaesth. 108 Suppl 1:ig2-5 Review

Brierley I, Shaw D (2016) Premortem interventions in dying children to optimise organ donation: an ethical analysis. I Med Ethics 42(7):424-8

Bundesärztekammer (2020) Richtlinie gemäß $§ 16$ Abs. 1 S. 1 Nr. 3 TPG zur ärztlichen Beurteilung nach $\$$ 9a Abs. 2 Nr. 1 TPG (RL BäK Spendererkennung). Dtsch Arztebl 2020; 117(35-36): A-1650/B-1414 
Bundesministerium für Justiz und Verbraucherschutz (2021) Transplantationsgesetz in der Fassung der Bekanntmachung vom 4. September 2007 (BGBl. I S. 2206), das zuletzt durch Artikel 19a des Gesetzes vom 3. Juni 2021 (BGBI. I S. 1309) geändert worden ist. URL: https://www.gesetze-iminternet.de/tpg/BJNR263100997.html (aufgerufen am 23.07.2021)

Burns IP, Sellers DE, Meyer EC et al. (2014) Epidemiology of death in the PICU at five U.S. teaching hospitals. Crit Care Med 42(9):2101-8

Commitee on Bioethics (2013) Ethical controversies in organ donation after circulatory death. Paediatrics 131(5):1021-6

European Society for Paediatric \& Neonatal Intensive Care Society Standards for End of Life Care including Organ/Tissue Donation. URL: https://espnic-online.org/Education/Standards-and-Guidelines

Eurotransplant 2021-https://statistics.eurotransplant.org/index.php?search_type=donors\&search_ organ $=\&$ search_region=\&search_period=2020\&search_characteristic=\&search_text=\&search_ collection=

Gelbart B (2017) Challenges of paediatric organ donation I Paediatr Child Health 53(6):534-539. Review Hahnenkamp K, Beckmann M, Buchardi $\mathrm{H}$ et al. (2016) Entscheidungen bei potentiellen Organspendern. Anästh Intensivmed 57:152-154

Hawkins KC, Scales A, Murphy P et al. (2018) Current status of paediatric and neonatal organ donation in the UK. Arch Dis Child 103(3):210-215

Janssens U, Burchardi H, Duttge G et al. (2013) Therapiezieländerung und Therapiebegrenzung in der Intensivmedizin. Positionspapier der Sektion Ethik der DIVI. Anaesthesist 62(1):47-52

Jöbges S, Denke C, Kumpf 0 et al. (2019) Gesprächsführung mit Angehörigen. Medizinische Klinik Intensivmedizin und Notfallmedizin 114(2): p. 122-127

Kentish-Barnes N, Duranteau J, Montlahuc C et al. (2017) Clinicians' Perception and Experience of Organ Donation From Brain-Dead Patients. Crit Care Med 45(9):1489-1499

Kentish-Barnes N, Cohen-Solal Z, Souppart V et al. (2019) Being Convinced and Taking Responsibility: A Qualitative Study of Family Members' Experience of Organ Donation Decision and Bereavement After Brain Death. Crit Care Med 47(4):526-534

Kotloff RM, Blosser S, Fulda G/ et al. (2015) Management of the Potential Organ Donor in the ICU: Society of Critical Care Medicine/American College of Chest Physicians/Association of Organ Procurement Organizations Consensus Statement. Crit Care Med 43(6):1291-325

Nakagawa TA, Shemie SD, Dryden-Palmer K et al. (2018) Parshuram CS, Brierley J.: Organ Donation Following Neurologic and Circulatory Determination of Death. Pediatr Crit Care Med 19(8S Suppl 2): S. 26-S32

Neitzke G, Burchardi H, Duttge G et al. (2016) Grenzen der Sinnhaftigkeit von Intensivmedizin. Positionspapier der Sektion Ethik der DIVI. Med Klin Intensivmed Notfmed.111(6):486-92

Neitzke G, Rogge A, Lücking KM et al. (2019) Entscheidungshilfe bei erweitertem intensivmedizinischem Behandlungsbedarf auf dem Weg zur Organspende. Positionspapier der Sektion Ethik und der Sektion Organspende und -transplantation der Deutschen Interdisziplinären Vereinigung für Intensiv- und Notfallmedizin (DIVI) unter Mitarbeit der Sektion Ethik der Deutschen Gesellschaft für Internistische Intensivmedizin und Notfallmedizin (DGIIN). Med Klin Intensivmed Notfmed 114(4):319-326

Rodrigue JR, Cornell DL, Howard RI (2008) Pediatric organ donation: what factors most influence parents' donation decisions? Pediatr Crit Care Med 9(2):180-5

Schöne-Seifert B, Prien T, Rellensmann G et al. (2011) Behandlung potenzieller Organspender im Präfinalstadium. Dtsch Arztebl 108(40): A-2080 